\title{
A Interatividade na Aprendizagem de Geometria Descritiva Através do Software HyperCAL ${ }^{3 D}$
}

\author{
The Interactivity in Descriptive Geometry Learning Through HyperCAL ${ }^{3 D}$ Software
}

BIDONE, Kelly; Mestranda; Universidade Federal do Rio Grande do Sul

kelly.bidone@gmail.com

GUGLIANO, Bruna Ferreira; Mestre; Universidade Federal do Rio Grande do Sul

brunafgugliano@gmail.com

SILVA, Régio Pierre da; Doutor; Universidade Federal do Rio Grande do Sul

regio@ufrgs.br

TEIXEIRA, Fábio Gonçalves; Doutor; Universidade Federal do Rio Grande do Sul

fabiogt@ufrgs.br

SILVA, Tânia Luisa Koltermann da; Doutora; Universidade Federal do Rio Grande do Sul

tania.koltermann@ufrgs.br

\section{Resumo}

Este artigo apresenta uma análise descritiva das formas de interação encontradas no software HyperCAL ${ }^{3 D}$. Os critérios de análise utilizados foram os tipos de interação propostos por Moreno e Mayer (2007) e Sims (1997), identificados com a realização de revisão bibliográfica. Foi elaborado um quadro comparativo com os tipos de interação para ambientes educacionais e funcionalidades do software em questão, considerando a relação dos conceitos de design de interação e de interatividade com o contexto de ensino-aprendizagem. A pesquisa apresenta também uma proposta de questionário a ser realizada com alunos, a fim de verificar os resultados do quadro comparativo. Como conclusão, o artigo indica que, por apresentar a maior parte dos tipos de interatividade levantados, o software favorece a eficácia da aprendizagem dos alunos de Geometria Descritiva, constituindo importante ferramenta de apoio ao trabalho desenvolvido em sala de aula, entretanto, são apontadas algumas formas de interatividade que ainda podem ser aperfeiçoadas.

Palavras Chave: design de interação; geometria descritiva; interatividade.

\begin{abstract}
This article presents a descriptive analysis of the interaction forms found in the HyperCAL ${ }^{3 D}$ software. The analysis criteria used were the types of interaction proposed by Moreno and Mayer (2007) and Sims (1997), identified through a bibliographic review. A comparative table was drawn up with the interaction types for educational environments and software functionalities, considering the relationship between the concepts of interaction design and interactivity with the learning context. The research also presents a proposal of a questionnaire to be carried out with the students, in order to verify the results of the comparative table. As a conclusion, the article indicates that, because it presents most of the types of interactivity raised, the software favors the
\end{abstract}


efficacy of the teaching of Descriptive Geometry, constituting an important tool to support the work developed in the classroom, however, they are pointed out some forms of interactivity that can still be improved.

Keywords: interaction design; descriptive geometry; interactivity.

\section{Introdução}

Sabe-se que a performance do aluno pode ser prejudicada quando a carga informacional sobrepõe a habilidade deste de compreendê-la. A frustração causada pelo excesso dessa carga leva ao esquecimento de detalhes importantes, a não absorção do conteúdo e até ao abandono da tarefa (WHITENTON, 2016). No caso das disciplinas de Geometria Descritiva, notou-se que o próprio esforço para visualizar as representações causava prejuízos no momento da aprendizagem, sendo necessária a criação de um ambiente que prestasse auxílio na concepção das formas em três dimensões (TEIXEIRA et al, 2015).

$\mathrm{O}$ aplicativo HyperCAL ${ }^{3 D}$ foi desenvolvido por um grupo de pesquisa, em uma instituição federal de ensino superior, a partir da constatação do grande esforço cognitivo feito pelos alunos das disciplinas de Geometria Descritiva "durante o processo de transposição de uma representação bidimensional para a tridimensional e vice-versa" (TEIXEIRA et al, 2015, p. 81).

Para Tarouco et al (2009), as tecnologias utilizadas nos processos de ensino e aprendizagem devem evitar um uso passivo por parte dos alunos, implementando funções que permitam a exploração e o engajamento ativo nos ambientes virtuais. A partir da constatação dos objetivos do aplicativo HyperCAL ${ }^{3 D}$, segundo Teixeira e Santos (2013, p. 2), nota-se que a ferramenta possibilita esse aprendizado ativo ao:

[...] reduzir o grau de abstração necessário para aprender os conceitos básicos; aproveitar os conhecimentos prévios dos estudantes; garantir o entendimento dos processos bidimensionais e os seus correspondentes tridimensionais e estimular o processo de aprendizagem a partir de problemas concretos com aplicações práticas.

A palavra "interativo", no contexto das ferramentas de aprendizagem baseadas em computador, está associada a melhores experiências, aprendizagem mais ativa, maior interesse e motivação dos alunos (SIMS, 1999). Desta forma, percebe-se uma preocupação em melhorar o aprendizado do aluno de Geometria Descritiva através do uso das tecnologias gráficas e da interação dos alunos com as mesmas. Ou seja: o aluno pode não somente visualizar as estruturas em um ambiente virtual, mas também construí-las e transformá-las. Esse fato vai ao encontro da definição dos objetivos do design de interação feita por Preece et al: "criar experiências que melhorem e estendam a maneira como as pessoas trabalham, se comunicam e interagem" (2005, p. 28). Ao dar suporte a uma atividade educacional, o HyperCAL ${ }^{3 D}$ pode ser considerado um produto interativo.

O presente artigo tem como objetivo analisar as formas de interação possíveis a partir da interface do sistema HyperCAL ${ }^{3 D}$, a fim de identificar como a interação com a ferramenta oferece suporte ao aprendizado dos conteúdos de sala de aula. Para isso, foram pesquisados conceitos de design de interação e de interatividade, relacionando-os com o contexto de ensino-aprendizagem. Logo após, foi elaborado um quadro comparativo com tipos de interação para ambientes educacionais e funcionalidades do software em questão, baseado no referencial teórico 
apresentado a seguir. Considerando que a análise foi feita a partir do ponto de vista dos pesquisadores, a fim de verificar os resultados, a pesquisa prevê a aplicação de um questionário com alunos das disciplinas de Geometria Descritiva de cursos de graduação, os quais utilizam o software analisado.

\section{Sobre o Design de Interação}

Os ambientes virtuais possibilitam o uso de diversos recursos de hipermídia para apresentar uma informação, como o uso de som, imagem, vídeo e acesso a links externos. A forma como esses recursos são apresentados e acessados é definida pelo designer de interação, que planeja a interatividade por meio de uma interface (PORTUGAL, 2013).

O design de interação trata, portanto, do comportamento de uma interface (produto, ou serviço), porém sempre relacionando-o com a tarefa dos indivíduos que a utilizam, a fim de facilitá-la.

Sua finalidade é promover a comunicação e a interação entre dois ou mais seres humanos ou, em um grau menor, entre um humano e uma entidade artificial capaz de reagir de alguma maneira, como um computador, um celular ou um aplicativo digital (SAFFER, 2010, p. 22).

Cabe apresentar dois conceitos que surgem na discussão sobre design de interação e que, muitas vezes, são apresentados como sinônimos do mesmo: a interação humano-computador (IHC) e a interatividade. No caso da primeira, de acordo com Preece et al (2005), trata-se de uma disciplina englobada pelo design de interação, da mesma forma que as ciências sociais, cognitivas, ergonômicas, etc. Seu foco encontra-se no desenvolvimento, implementação e avaliação das interfaces interativas em si, em termos mais quantitativos.

Já "interatividade" é um conceito voltado especificamente para a questão tecnológica. Para Lemos (1997), o termo interatividade refere-se substancialmente ao contexto eletrônicodigital, ligado às novas mídias. A interatividade seria, portanto, uma interação digital, enquanto as que não necessitam de um apoio técnico seriam interações sociais. O termo "interação" por si só pode ser usado em diversas áreas para referir-se a quaisquer elementos que interagem entre si. $\mathrm{Na}$ educação, por exemplo, podem-se perceber três tipos de interação que independem dos meios técnicos (MOORE, 1989): a interação aluno-conteúdo, a aluno-professor e a aluno-aluno.

Com a educação a distância explorando cada vez mais as tecnologias digitais, Hillman et al (1994) chamam a atenção para a necessidade de um novo tipo de interação: a do aluno-interface. Nela, o aluno interage com um meio tecnológico para, então, poder interagir com os demais elementos (conteúdo, professor e demais alunos). Os autores salientam que uma interação efetiva entre aluno-interface requer que o aluno compreenda os procedimentos para lidar com as ferramentas. Por isso,

ao criar uma instrução a ser transmitida pelas tecnologias de comunicação, o designer instrucional deve ter em mente que o usuário não pode lidar com o conteúdo da instrução caso ele ou ela não consiga interagir inicialmente com a interface (1994, p. 35). 
Outros tipos de interação encontram-se na literatura, como a auto-interação, a interação vicária, aluno-outros, aluno-ambiente, etc (MATTAR, 2014) ${ }^{1}$, porém, o presente artigo pretende abordar somente a interação entre o aluno e as ferramentas apresentadas pela interface do HyperCAL ${ }^{3 D}$, como será apresentado na próxima seção.

\section{O HyperCAL ${ }^{3 D}$ e a Interatividade}

O software HyperCAL ${ }^{3 D}$ (Figura 1) permite a criação e a edição de modelos tridimensionais a partir de coordenadas de vértices e conectividades de elementos geométricos, como arestas e faces (HyperCAL ${ }^{3 \mathrm{D}}$ ).

Figura 1 - Interface do software HyperCAL ${ }^{3 D}$.

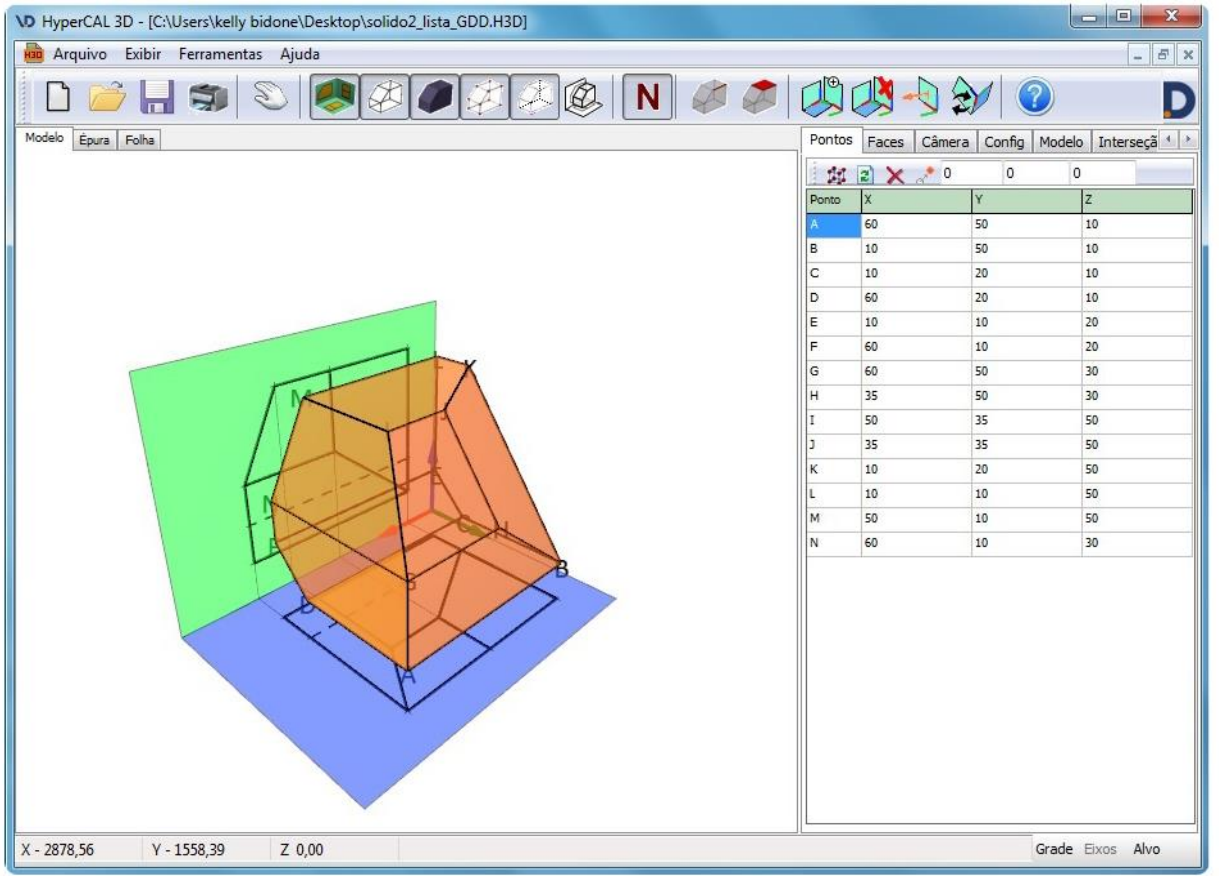

Fonte: os autores.

A interface do software (Figuras 1 e 2) apresenta barra de menu (1), barra de ferramentas de manipulação de arquivos, visualização e aparência do modelo, mudança do sistema de referência e ajuda (2), área do modelo (3), área de edição e configuração do modelo e da folha de impressão (4), coordenadas (5), barra de ajuda (6) e configuração de visibilidade da grade, eixo ou alvo (7). O material de ajuda compreende conteúdo explicativo da finalidade e possibilidades de utilização do software e suas ferramentas (Figura 3).

\footnotetext{
${ }^{1}$ A auto-interação diz respeito aos processos cognitivos e metacognitivos dos alunos. A interação vicária refere-se à aprendizagem baseada na observação, absorção e reflexão das interações realizadas pelos colegas e professores. A interação aluno-outros abarca todas as interações fora do âmbito do curso, podendo ser com especialistas, a comunidade, redes, mídias sociais, a família, entre outros. A interação aluno-ambiente existe na realização de visitas à locais externos ou realização de trabalho fora do ambiente virtual (MATTAR, 2014).
} 
Figura 2 - Áreas da interface do software HyperCAL ${ }^{3 \mathrm{D}}$.

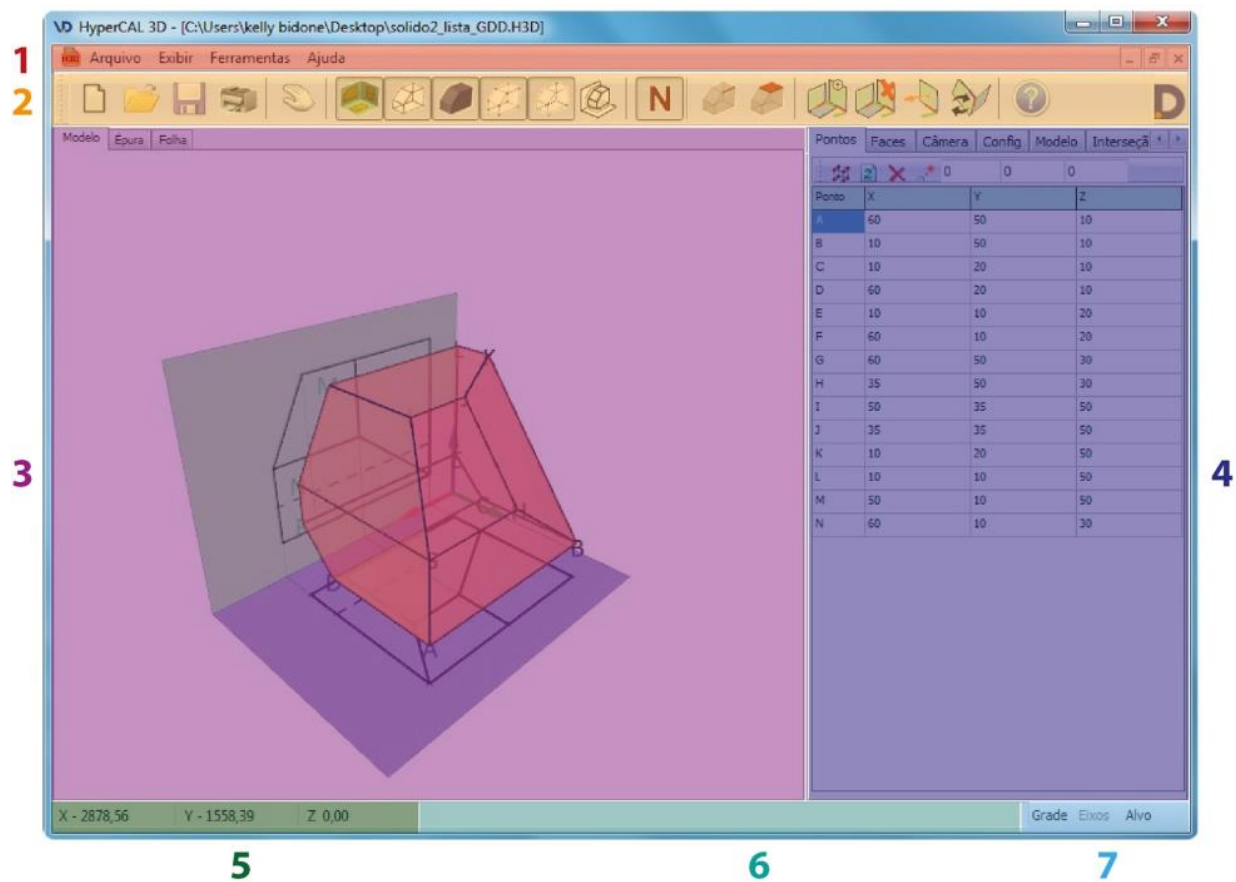

Fonte: os autores.

Figura 3 - Interface do material de ajuda do software HyperCAL ${ }^{3 D}$.

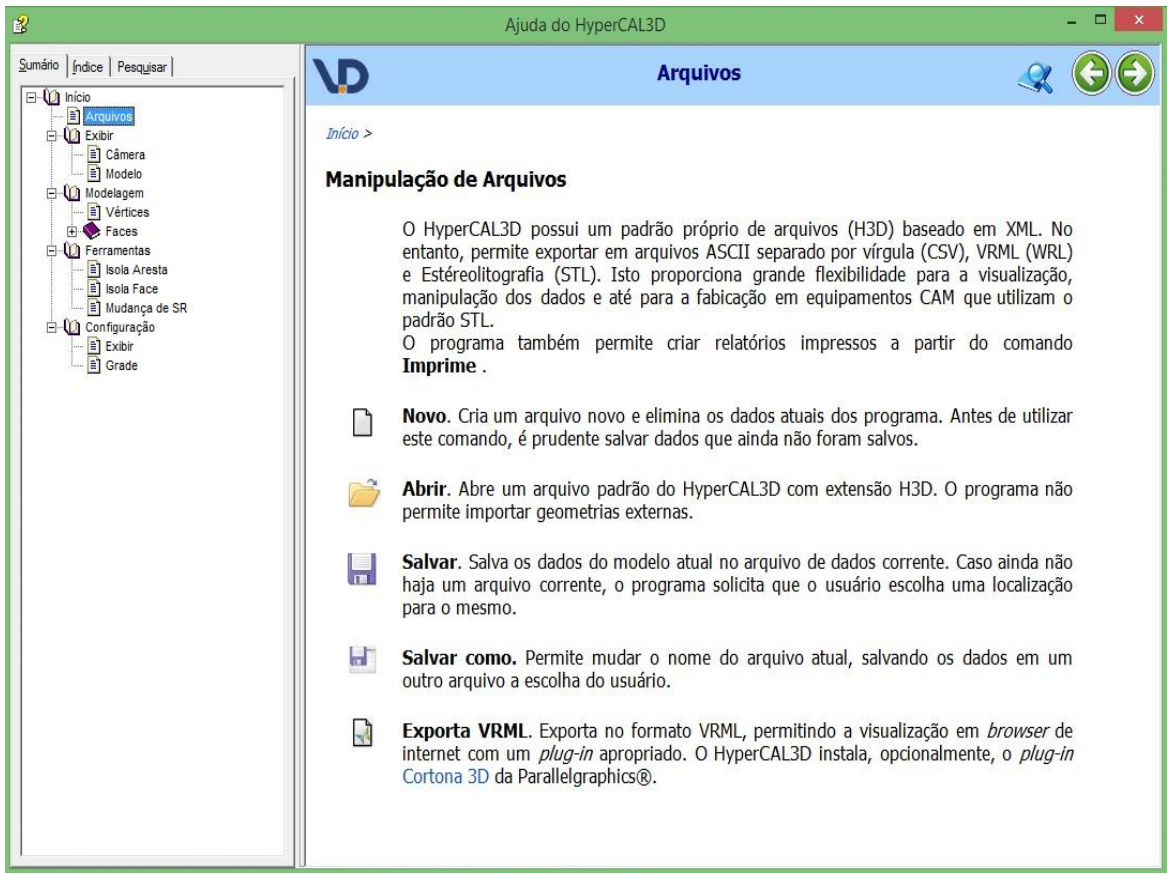

Fonte: os autores. 
A interatividade de um produto pode apresentar-se de várias maneiras. Neste item pretende-se analisar a aplicação dos diferentes tipos de interatividade no software HyperCAL ${ }^{3 D}$.

Moreno e Mayer (2007) apresentam cinco funções que podem ser encontradas nas interfaces interativas para a educação. São elas: dialogar, controlar, manipular, pesquisar e navegar. No quadro abaixo as funcionalidades do HyperCAL ${ }^{3 \mathrm{D}}$ são analisadas de acordo com esses tipos de interatividade. Nele são apresentadas as descrições dos conceitos e sua aplicação prática no aplicativo.

Quadro 1 - Funções interativas e a aplicação no software HyperCAL ${ }^{3 D}$.

\begin{tabular}{lll}
\hline Função & Descrição & Aplicação no HyperCAL \\
\hline Dialogar & $\begin{array}{l}\text { Aluno recebe perguntas e } \\
\text { respostas ou feedback para a seu } \\
\text { input. }\end{array}$ & $\begin{array}{l}\text { Apresenta feedback para os inputs do usuário, porém não } \\
\text { quando dados inapropriados são inseridos. }\end{array}$ \\
\hline Controlar & $\begin{array}{l}\text { Aluno determina o ritmo e/ou a } \\
\text { ordem de apresentação. }\end{array}$ & $\begin{array}{l}\text { O usuário é capaz de alternar as telas "modelo", "épura" e } \\
\text { "folha" (Figura 2 - área 3), além de mover-se entre as } \\
\text { abas de edição e configuração do modelo e demais } \\
\text { menus, de acordo com a necessidade, a qualquer } \\
\text { momento. O conteúdo de ajuda também apresenta esse } \\
\text { tipo de interação. }\end{array}$ \\
\hline
\end{tabular}

Manipular Aluno define parâmetros para uma simulação, pode aumentar ou diminuir o zoom e movimentar objetos pela tela.
O aluno pode manipular modelos já existentes ou criar um novo, a partir de parâmetros estipulados pelo mesmo. Também é possível o ajuste de zoom (aproximação ou distanciamento) e movimento (vertical, horizontal, rotação).

\begin{tabular}{|c|c|c|}
\hline Pesquisar & $\begin{array}{l}\text { Aluno encontra um novo material } \\
\text { ao inserir uma consulta, receber } \\
\text { opções e selecionar uma } \\
\text { alternativa. }\end{array}$ & Não apresenta. \\
\hline Navegar & $\begin{array}{l}\text { Aluno é capaz de mover-se para } \\
\text { diferentes áreas do conteúdo, } \\
\text { selecionando a partir de várias } \\
\text { fontes de informação disponíveis. }\end{array}$ & $\begin{array}{l}\text { Apresenta no conteúdo de ajuda, no qual o usuário é } \\
\text { capaz de navegar no material a partir do sumário, índice e } \\
\text { pesquisa, links presentes nos textos, além dos botões de } \\
\text { avanço e retrocesso do conteúdo, os quais possibilitam o } \\
\text { controle da apresentação do material pelo aluno. }\end{array}$ \\
\hline
\end{tabular}

Fonte: adaptado de Moreno e Mayer (2007).

Roderick Sims (1997) apresenta algumas formas de interatividade que podem ser utilizadas em benefício do aprendizado do aluno nas interfaces. Esses conceitos podem ser aplicados na avaliação dos projetos de materiais educacionais multimídia (MATTAR, 2014). Da mesma forma realizada acima, no quadro seguinte as funcionalidades do HyperCAL ${ }^{3 \mathrm{D}}$ são analisadas de acordo com os tipos de interatividade citados por Sims (1997). 
Quadro 2 - Tipos de interatividade e a aplicação no software HyperCAL ${ }^{3 D}$.

\begin{tabular}{lll}
$\begin{array}{l}\text { Tipos de } \\
\text { interatividade }\end{array}$ & Descrição & Aplicação no HyperCAL $^{\text {BD }}$ \\
\hline Objetos & $\begin{array}{l}\text { Resposta audiovisual a cliques em objetos } \\
\text { (botões, pessoas, coisas). }\end{array}$ & $\begin{array}{l}\text { A interação com botões e elementos do modelo } \\
\text { apresentam resposta visual a cliques, porém, não } \\
\text { apresentam áudio. }\end{array}$ \\
\hline
\end{tabular}

\begin{tabular}{lll}
\hline Linear & $\begin{array}{l}\text { Usuário é capaz de mover-se de forma } \\
\text { linear (para frente ou para trás) através do } \\
\text { material instrucional. }\end{array}$ & $\begin{array}{l}\text { Apresenta no conteúdo de ajuda, através de } \\
\text { botões de avanço e retrocesso do conteúdo. }\end{array}$ \\
\hline Suporte & $\begin{array}{l}\text { O material oferece suporte, podendo } \\
\text { apresentar-se como de simples mensagens } \\
\text { de ajuda a tutoriais complexos. }\end{array}$ & $\begin{array}{l}\text { Apresenta detalhado conteúdo de ajuda. } \\
\text { Apresenta mensagens de ajuda durante a } \\
\text { realização de tarefas apenas em alguns comandos } \\
\text { (Figura 2 - área 6). Não apresenta tutoriais. }\end{array}$
\end{tabular}

\begin{tabular}{lll}
\hline Atualização & $\begin{array}{l}\text { Atualizações ou feedbacks individualizados } \\
\text { no diálogo estabelecido entre o aluno e o } \\
\text { conteúdo gerado pelo sistema. }\end{array}$ & $\begin{array}{l}\text { Apresenta parcialmente. Alguns comandos } \\
\text { apresentam feedback na barra de ajuda (Figura 2- } \\
\end{array}$ \\
$\begin{array}{ll}\text { área 6). Ao clicar em determinadas ferramentas, o } \\
\text { sistema indica a próxima ação para completar o } \\
\text { comando. }\end{array}$
\end{tabular}

\begin{tabular}{lll}
\hline Construção & $\begin{array}{l}\text { Realização da tarefa depende da } \\
\text { manipulação de objetos. }\end{array}$ & $\begin{array}{l}\text { Apresenta. O software é baseado na construção e } \\
\text { edição de modelos tridimensionais. }\end{array}$ \\
\hline Refletida & $\begin{array}{l}\text { Respostas vão além de "certo" ou "errado". } \\
\text { Possibilidade de comparação da resposta do } \\
\text { aluno com a de outros usuários ou de } \\
\text { especialistas. }\end{array}$ & Não apresenta. \\
\hline Simulação & $\begin{array}{l}\text { Relacionada à interatividade de construção, } \\
\text { a ação do aluno influencia na simulação de } \\
\text { objetos ou situações. }\end{array}$ & \\
\hline Hiperlinks & $\begin{array}{l}\text { Acesso a uma diversidade de informaçães } \\
\text { através da navegação por hiperlinks. }\end{array}$ & Não apresenta. \\
\hline Contextual & $\begin{array}{l}\text { Este conceito combina e estende-se aos } \\
\text { vários tipos de interatividade. O aluno } \\
\text { narticipa ativamente e em contexto similar } \\
\text { ao mundo real. }\end{array}$ & $\begin{array}{l}\text { A construção e simulação dos modelos assemelha- } \\
\text { se ao que é realizado em sala de aula. }\end{array}$ \\
\hline $\begin{array}{l}\text { Virtual } \\
\text { imersiva }\end{array}$ & $\begin{array}{l}\text { Fornece um ambiente interativo no qual o } \\
\text { aluno é projetado em um mundo completo } \\
\text { gerado por computador, o qual responde ao } \\
\text { movimento e as ações do usuário. }\end{array}$ \\
\hline
\end{tabular}

Fonte: adaptado de Sims (1997). 
Para a adequada implementação desses tipos de interação, Sims (1997) destaca a necessidade do equacionamento entre a compreensão do aluno, a otimização das capacidades da engenharia de software, a prática rigorosa do design instrucional e a aplicação de interfaces gráficas adequadas. Assim, seria possível o desenvolvimento de aplicações interativas, educativas e eficazes.

\section{Discussão}

Moreno e Mayer (2007) seguem a visão de que a aprendizagem acontece através da construção do conhecimento em ambientes interativos que favoreçam a comunicação alunosistema, nos quais os alunos participam ativamente desse processo. Na mesma linha, Roderick Sims (1997) coloca a interação como algo que é intrínseco ao sucesso e eficácia da prática educacional. Sendo assim, entende-se que o software HyperCAL ${ }^{3 D}$ está alinhado à essas ideias, visto que apresenta a maior parte das formas de interatividade analisadas.

O software tem por base as interatividades de construção e simulação (SIMS, 1997), pois, para atingir o objetivo de construir o modelo tridimensional, o aluno precisa manipular as ferramentas do software utilizando o conhecimento das disciplinas de Geometria Descritiva.

Contudo, o software ainda pode ser aperfeiçoado em termos de interação. Notou-se que a interação na forma de diálogo (MORENO; MAYER, 2007) poderia ser aprimorada, dado que quando o usuário insere algum caractere nos campos de edição do modelo, o sistema não responde a possíveis equívocos.

A possibilidade de interação por pesquisa, sugerida por Moreno e Mayer (2007), não é apresentada, provavelmente pelo fato de que o software não depende de conexão com a internet, porém, assim como acontece com o material de ajuda, poderiam ser vinculados outros materiais, como tutoriais, por exemplo. Da mesma forma em relação à interatividade através de hiperlinks (SIMS, 1997).

As mensagens de ajuda indicadas por Sims (1997), identificadas no software durante a realização de poucos comandos, poderiam ser estendidas às demais operações úteis aos usuários durante a construção dos modelos.

Entende-se que a interatividade refletida (SIMS, 1997) não é verificada no software por fugir ao propósito do mesmo, uma vez que não são propostos exercícios que envolvam perguntas e respostas.

\section{Questionário com os Usuários}

A próxima etapa desta pesquisa contará com um questionário online feito com os usuários (alunos) que já utilizaram o software nas disciplinas de Geometria Descritiva. A aplicação do questionário se dará através da plataforma GoogleForms, que possui as opções de escala Likert (LEITE, 2008), necessárias para esse procedimento. Para Flick (2009), as condições para que uma pesquisa seja feita de forma online são: os pesquisadores devem estar habituados a utilizar um computador de forma abrangente, possuindo experiência no uso de softwares além dos editores de texto. Os pesquisadores devem, também, gostar de trabalhar online e ter acesso frequente à internet, estando familiarizados com as tecnologias de informação e comunicação. Para o autor, após cumpridas essas condições, se deve ponderar se a pesquisa online é a opção mais adequada, considerando o públicoalvo. Sendo o público-alvo estudantes de Design que tem no computador sua ferramenta de trabalho e estudo, considera-se que um questionário online tenha boa aceitação entre a amostra. 
As questões serão feitas com foco nas funções interativas (MORENO; MAYER 2007) e nos tipos de interatividade (SIMS, 1997), perguntando aos usuários sobre sua percepção a respeito desses recursos. Uma lista contendo o título e descrição de cada função/tipo de interatividade será apresentada juntamente com uma escala Likert (LEITE, 2008), na qual o/a respondente poderá assinalar a relevância percebida por ele/ela a respeito de cada item. A escala apresentará opções de 1 ao 5, sendo 1 "não apresenta", 3 "não sei avaliar" e 5 "apresenta". A figura 4 ilustra um modelo de questão.

Figura 4 - Questionário de avaliação da interatividade do software HyperCAL ${ }^{3 D}$.

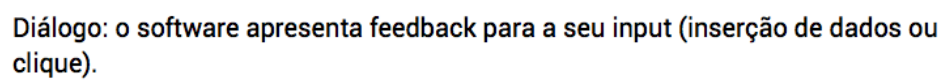
clique).

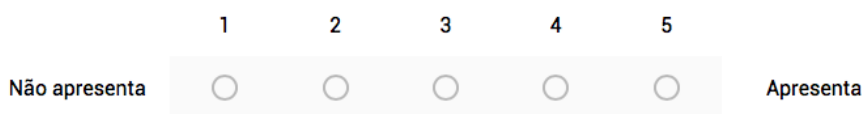

Fonte: os autores.

\section{Considerações Finais}

O software HyperCAL ${ }^{3 \mathrm{D}}$ apresenta a maior parte dos tipos de interatividade indicados por Moreno e Mayer (2007) e Sims (1997), sendo assim, pode ser considerado interativo. De acordo com as teorias apresentadas, essa característica indica que o software favorece a eficácia da aprendizagem dos alunos de Geometria Descritiva, constituindo uma importante ferramenta de apoio ao trabalho desenvolvido em sala de aula.

A partir da próxima etapa, de questionário com usuários, será possível estabelecer quais pontos da interatividade podem ser aperfeiçoados para uma melhor experiência dos alunos, além de verificar os resultados da pesquisa realizada até então. $O$ aprimoramento do software com a exploração mais aprofundada ou inclusão de diferentes tipos de interatividade deve aumentar a atratividade do mesmo, visto que a utilização desse tipo de ferramenta envolve o aluno em um processo de aprendizagem mais ativo.

\section{Referências}

FLICK, Uwe. Introdução à Pesquisa Qualitativa. 3 ed. Porto Alegre: Artmed, 2009.

HILLMAN, Daniel CA; WILLIS, Deborah J.; GUNAWARDENA, Charlotte N. Learner-interface interaction in distance education: An extension of contemporary models and strategies for practitioners. American Journal of Distance Education, v. 8, n. 2, p. 30-42, 1994.

KUKULSKA-HULME, Agnes; SHIELD, Lesley. The keys to usability in e-learning Web sites. In: Networked Learning Conference. 2004. Disponível em: <http://www.networkedlearningconference.org.uk/past/nlc2004/proceedings/individual_papers/ kukulska_shield.htm>. Acesso em: 05 out. 2016.

LEITE, Francisco. Metodologia Científica: métodos e técnicas de pesquisa. Aparecida: Idéias \& Letras, 2008.

LEMOS, André. Anjos Interativos e Retribalização do Mundo: sobre interatividade e interfaces 
digitais, 1997. Disponível em: <http://www.facom.ufba.br/ciberpesquisa/lemos/interativo.pdf>. Acesso em: 07 out. 2016.

MATTAR, J. Design Educacional: educação a distância na prática. São Paulo: Artesanato Educacional, 2014.

MOORE, Michael G. Editorial: Three types of interaction. American Journal of Distance Education, v. 3, n. 2, p. 1-7, 1989.

MORENO, R.; MAYER, R. E. Interactive multimodal learning environments. Educational Psychology Review, v. 19, p. 309-326, 2007.

PREECE, Jennifer. ROGERS, Yvone. SHARP, Helen. Design de Interação: além da interação homemcomputador. Porto Alegre: Bookman, 2005.

PORTUGAL, C. Design, Educação e Tecnologia. Rio de Janeiro: Rio Books, 2013.

SAFFER, Dan. Designing for Interaction: creating innovative applications and devices. New Riders, 2010.

SIMS, Roderick. Interactivity: A Forgotten Art?. 1997. Disponível em: <http://www2.gsu.edu/ wwwitr/docs/interact/>. Acesso em: 08 out. 2016.

SIMS, Roderick. Interactivity on stage: strategies for learner-designer communication. Australian Journal of Educational Technology, v. 15, n. 3, p. 257-272, 1999.

TAROUCO, Liane Margarida Rockenbach et al. Multimídia Interativa: princípios e ferramentas. RENOTE, v. 7, n. 1, 2009.

TEIXEIRA, Fábio Gonçalves; SANTOS, Sérgio Leandro dos. HyperCAL3D, Um sistema inovador para auxílio ao processo de ensino de geometria descritiva. Design \& Tecnologia, v. 6, p. 20-32, 2013.

TEIXEIRA, Fábio Gonçalves; SILVA, Tânia Luisa Koltermann; SILVA; Régio Pierre da; BRUNO, Fernando Batista. Experiências inovadoras em ensino e pesquisa da geometria descritiva. Revista Brasileira de Expressão Gráfica. Vol. 3, No. 3, 2015.

WHITENTON, Kathryn. Minimize Cognitive Load to Maximize Usability. Disponível em: <https://www.nngroup.com/articles/minimize-cognitive-load/>. Acesso em: 08 out. 2016. 